\title{
Directional Volume Growing for the Extraction of White Matter Tracts from Diffusion Tensor Data
}

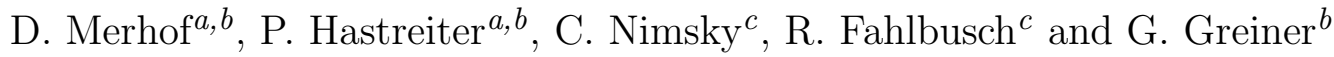 \\ ${ }^{a}$ Neurocenter, Dept. of Neurosurgery, University of Erlangen-Nuremberg, Germany \\ ${ }^{b}$ Computer Graphics Group, University of Erlangen-Nuremberg, Germany \\ ${ }^{c}$ Dept. of Neurosurgery, University of Erlangen-Nuremberg, Germany
}

\begin{abstract}
Diffusion tensor imaging measures diffusion of water in tissue. Within structured tissue, such as neural fiber tracts of the human brain, anisotropic diffusion is observed since the cell membranes of the long cylindric nerves restrict diffusion. Diffusion tensor imaging thus provides information about neural fiber tracts within the human brain which is of major interest for neurosurgery. However, the visualization is a challenging task due to noise and limited resolution of the data. A common visualization strategy of white matter is fiber tracking which utilizes techniques known from flow visualization. The resulting streamlines provide a good impression of the spatial relation of fibers and anatomy. Therefore, they are a valuable supplement for neurosurgical planning. As a drawback, fibers may diverge from the exact path due to numerical inaccuracies during streamline propagation even if higher order integration is used. To overcome this problem, a novel strategy for directional volume growing is presented which enables the extraction of separate tract systems and thus allows to compare and estimate the quality of fiber tracking algorithms. Furthermore, the presented approach is suited to get a more precise representation of the volume encompassing white matter tracts. Thereby, the entire volume potentially containing fibers is provided in contrast to fiber tracking which only shows a more restricted representation of the actual volume of interest. This is of major importance in brain tumor cases where white matter tracts are in the close vicinity of brain tumors. Overall, the presented strategy contributes to make surgical planning safer and more reliable.
\end{abstract}

Keywords: Diffusion Tensor Imaging, Volume Growing, Fiber Tracts

\section{INTRODUCTION}

Diffusion tensor imaging (DTI) is a recent MR acquisition technique that measures the random motion of water molecules known as Brownian motion. Within structured tissue, such as neural fiber tracts of the human brain, anisotropic diffusion is observed since the cell membranes of the long cylindric nerves restrict diffusion. DTI thus provides information about neural fiber tracts within the human brain which is of major interest for neurosurgery. However, the visualization is a challenging task due to noise and limited resolution of the data.

For each voxel, diffusion is represented by a second order tensor that is computed from the DTI data. These diffusion tensors are the basis for different visualization approaches. The techniques presented over the last years for visualizing DT data either extract just local information or require a simplification of the tensor field resulting in a scalar or vector field.

Several diffusion metrics have been presented such as fractional anisotropy (FA) or the barycentric space coordinates $c_{l}, c_{p}, c_{s}$ which characterize the shape of a diffusion tensor ( $l$ : linear, $p$ : planar, $s$ : spherical). Respective 2D slice images allow inspecting the local relation between a tumor and surrounding white matter. A promising approach in 3D is fiber tracking which basically utilizes streamline techniques known from flow visualization. It provides a much better impression of the spatial relation between fibers and space occupying 
lesions and is therefore a valuable supplement for neurosurgical planning. However, the streamline representation is in some respects misleading since a rendered streamline does not correlate with a single nerve cell. A technical problem of tracking algorithms is that errors accumulate with increasing distance to the seed points so that fibers drift away from the exact path even if higher order integration methods are used. Furthermore, the tracking result depends on the choice of the tracking parameters, which is especially true for regularized stochastic approaches. As a result of these influencing factors certain parts of the fiber tract may be missed. As a consequence, the underlying anatomy is not comprehensively represented. To cope with these limitations, a new strategy for directional volume growing is presented which enables to extract separate tract systems. This approach encompasses all anisotropic material and gives a more precise impression of the volume occupied by white matter, which makes surgical planning safer.

This paper is organized as follows: Section 2 provides a short overview about image acquisition. In Section 3 previous work on DTI visualization is described including remarks on open problems and limitations of the respective technique. Our new approach for directional volume growing is presented in Section 4. Experimental results using different datasets and a short discussion of the approach are presented in Section 5 . The conclusion in Section 6 summarizes the contribution of the presented approach.

\section{IMAGE ACQUISITION}

For the computation of diffusion tensors one reference image and at least six diffusion images are required. All images were acquired using a Siemens MR Magnetom Sonata Maestro Class 1.5 Tesla scanner equipped with a gradient system with a field strength of up to $40 \mathrm{mT} / \mathrm{m}$ (effective $69 \mathrm{mT} / \mathrm{m}$ ) and a slew rate of up to 200 $\mathrm{T} / \mathrm{m} / \mathrm{s}$ (effective $346 \mathrm{~T} / \mathrm{m} / \mathrm{s}$ ). The datasets consisted of $128 \times 128 \times 60$ voxels with a spatial resolution of $1.875 \times 1.875 \times 1.9 \mathrm{~mm}^{3}$.

DT image data: The diffusion images differed in the direction of the gradient that was applied. For each slice a total amount of seven images was created: One reference image was measured without any gradient direction which is equivalent to common $\mathrm{T} 2$ weighed MR data. The remaining six or alternatively twelve images were acquired using different gradient directions that correspond to the diffusion directions measured at that time. With respect to anatomical information, the reference image represents anatomical structures, the other images show diffusion properties.

DT imaging parameters: $\mathrm{TR}=9200, \mathrm{TE}=86 \mathrm{~ms}, \mathrm{~b}_{\text {high }}=1000 \mathrm{~s} / \mathrm{mm}^{2}, \mathrm{~b}_{\text {low }}=0 \mathrm{~s} / \mathrm{mm}^{2}$, field of view $240 \mathrm{~mm}, 1502 \mathrm{~Hz} / \mathrm{Px}$ bandwidth. The diffusion-encoding gradients for the six diffusion weighted images were directed along the following axes: $( \pm 1,1,0),( \pm 1,0,1)$ and $(1, \pm 1,0)$.
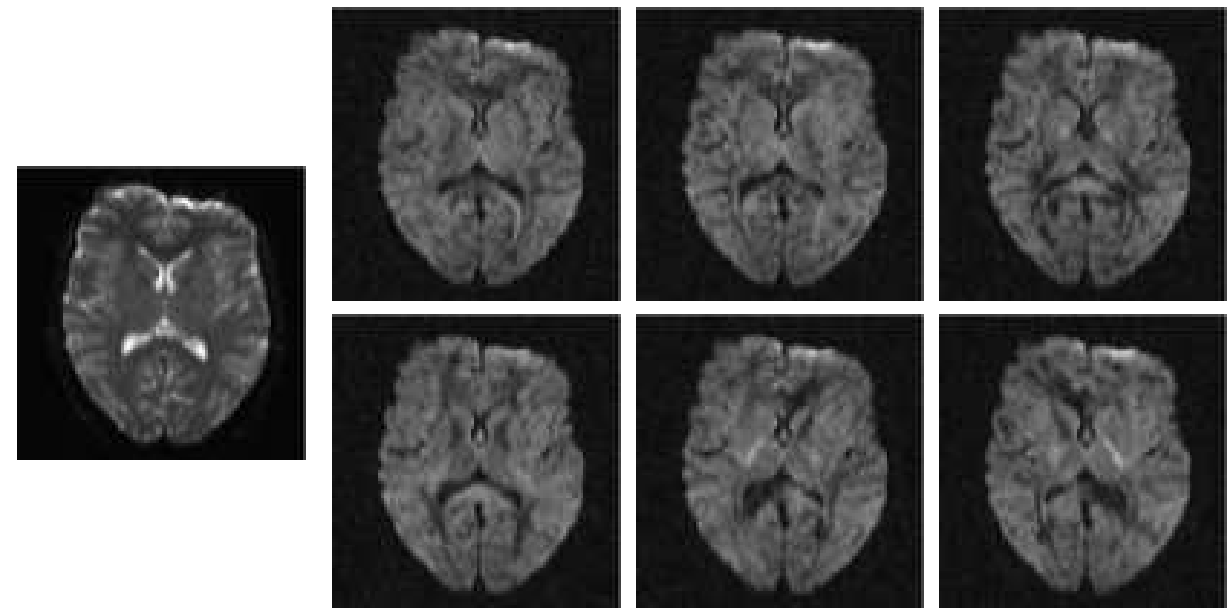

Figure 1. Diffusion tensor data. Left: Reference image. Right: Diffusion images for six different gradient directions. 


\section{VISUALIZATION APPROACHES}

The basis for all visualization approaches are second order diffusion tensors that are computed from the DTI data. For each voxel the corresponding diffusion tensor is derived by solving the Stejskal-Tanner equations system. $^{1}$ In case of six diffusion images an unambiguous solution for the equations system exists, otherwise a best fit solution has to be approximated. The resulting diffusion tensor $\mathbf{D}$ is a real Hermitian positive definite matrix, i.e. the matrix is symmetric with real and positive eigenvalues.

$$
\mathbf{D}=\left[\begin{array}{lll}
D_{x x} & D_{y x} & D_{z x} \\
D_{x y} & D_{y y} & D_{z y} \\
D_{x z} & D_{y} z & D_{z z}
\end{array}\right]
$$

If necessary, a continuous tensor field is reconstructed using trilinear interpolation. According to the findings of Kindlmann ${ }^{2}$ channel and matrix interpolation tend to track each other closely whereas eigenvalue interpolation only works well as long as the sampling density is sufficient. For this reason, we perform trilinear interpolation component-wise for each tensor entry to compute intermediate tensors. Alternatively, a B-spline-based approach $^{3,4}$ could be used to generate a continuous tensor field. This tensor field serves as basis for the different DTI visualization approaches which in general exploit the tensor information given by the respective eigenvalues and eigenvectors. In the following, the eigenvalues and eigenvectors of the diffusion tensor $\mathbf{D}$ are denoted by $\left(\lambda_{i}\right)_{i=1,2,3}$ with $\lambda_{1} \geq \lambda_{2} \geq \lambda_{3}$ and $\left(\vec{e}_{i}\right)_{i=1,2,3}$.

\subsection{Diffusion Metrics}

The visualization of high dimensional data such as tensor data is a challenging task. An approach to face this problem is to simplify the tensor data to a scalar diffusion metric. Different scalar measurements have been presented, ${ }^{5,6}$ two commonly used metrics are the Mean Diffusivity $\langle\mathbf{D}\rangle$ which characterizes the overall amount of diffusion and the Fractional Anisotropy FA(D) which is related to the presence of oriented structures and describes a directional bias.

$$
\langle\mathbf{D}\rangle=\frac{\operatorname{Trace}(\mathbf{D})}{3}=\frac{\sum_{i} \lambda_{i}}{3} \quad \operatorname{FA}(\mathbf{D})=\sqrt{\frac{3}{2}} \sqrt{\frac{\left(\lambda_{1}-\langle\mathbf{D}\rangle\right)^{2}+\left(\lambda_{2}-\langle\mathbf{D}\rangle\right)^{2}+\left(\lambda_{3}-\langle\mathbf{D}\rangle\right)^{2}}{\lambda_{1}^{2}+\lambda_{2}^{2}+\lambda_{3}^{2}}}
$$

An approach presented by Westin et al. ${ }^{7}$ characterizes the shape of a tensor by assigning coordinates in a barycentric space. This space is spanned by three basis tensor shapes which are linear, planar and spherical diffusion. In general, a diffusion tensor may be geometrically represented by an ellipsoid whereas the axes of the ellipsoid are oriented along the three perpendicular eigenvectors of the tensor. The length of the semiaxes is proportional to the square root of the respective eigenvalue. In the linear case, the tensor ellipsoid is cigar shaped and diffusion in direction of the eigenvector corresponding to the largest eigenvalue dominates (i.e. $\lambda_{1} \gg \lambda_{2} \approx \lambda_{3}$ ). In the planar case the diffusion ellipsoid is flat which corresponds to diffusion in a plane spanned by the eigenvectors with respective eigenvalues $\lambda_{1}$ and $\lambda_{2}$ (i.e. $\lambda_{1} \approx \lambda_{2} \gg \lambda_{3}$ ). In the spherical case, the shape of the tensor is a sphere and no preferred diffusion direction exists (i.e. $\lambda_{1} \approx \lambda_{2} \approx \lambda_{3}$ ). Accordingly, the barycentric coordinates of linear $c_{l}$, planar $c_{p}$ and spherical $c_{s}$ diffusion are defined as follows:

$$
\begin{gathered}
c_{l}=\frac{\lambda_{1}-\lambda_{2}}{\lambda_{1}+\lambda_{2}+\lambda_{3}} \\
c_{p}=\frac{2\left(\lambda_{2}-\lambda_{3}\right)}{\lambda_{1}+\lambda_{2}+\lambda_{3}} \\
c_{s}=\frac{3 \lambda_{3}}{\lambda_{1}+\lambda_{2}+\lambda_{3}} \\
c_{l}+c_{p}+c_{s}=1
\end{gathered}
$$

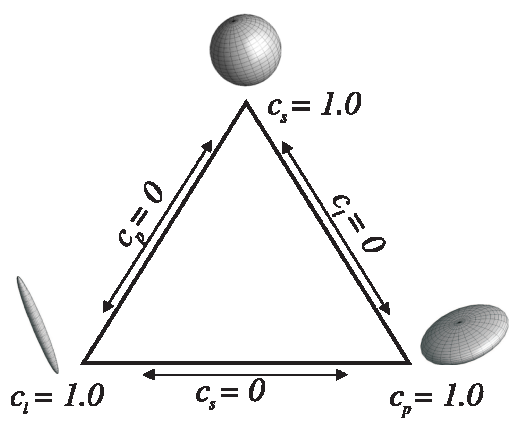




\subsection{Tensor Glyphs}

The simplification of a tensor to a scalar metric reduces the amount of information contained in the tensor. A possibility to show the entire information of the second order tensors is to use glyphs. Several shapes have been presented for representing tensors using glyphs. ${ }^{8,9}$ A popular approach is to use ellipsoids whereas the axes of the ellipsoid correlate with the directions of the eigenvectors and are scaled according to the corresponding eigenvalues. An advantage of tensor ellipsoids is that they can be rendered in real-time using graphics hardware. ${ }^{10}$ An even better shape for tensors are superquadric tensor glyphs ${ }^{11}$ which provide a better and unambiguous spatial understanding. However, superquadrics are computationally much more expensive and are not available in real-time.

The advantage of glyph-based techniques is that the entire tensor information is visualized. However, these visualizations are difficult to interpret as no global connectivity information is provided. Consequently, a combination with other visualization techniques is recommended to obtain a meaningful representation. In addition, clipping or slice views of glyphs are necessary to avoid an overloading and cluttering of the 3D information.

\subsection{Vector Field Approaches}

Alternatively, the tensor field can be simplified to a vector field consisting of the principal eigenvectors (corresponding to the largest eigenvalue) of each tensor. This approach is based upon the assumption that the principal eigenvector points in the direction of main diffusion. The resulting vector field thus reflects both orientation and structure of fibrous tissue.

A popular approach to visualize the field of principal eigenvectors is to map the vectors to color. This is achieved by assigning RGB colors to the x,y,z-coordinates of each normalized principal eigenvector. In addition, a diffusion metric such as FA is used to attenuate brightness, i.e. areas with low FA values are visualized darker. An alternative approach are hue-balls ${ }^{12}$ which color encode the deflection of a predefined vector after multiplication with the tensor using a grey-scale or color-scale sphere. Respective $2 \mathrm{D}$ slice images provide information about the location of white matter as well as directional information indicated by color.

However, these approaches provide no real 3D sight of the vector field. This is achieved by fiber tracking techniques which in general are based upon streamline tracing known from flow visualization. Streamline tracing is performed by computing a parametric 3D curve $p(t)$ through linear integration of the principal eigenvector where $t$ is the parameter of the curve and $\vec{e}_{1}(s)$ is the principal unit eigenvector as a function of position:

$$
p(t)=\int_{0}^{t} \vec{e}_{1}(s) d s
$$

Fiber tracking algorithms often utilize thresholds, angle criterions, regularization techniques and local filters to improve the results. However, most of the algorithms have a number of features in common: Regions of interest (ROIs) are specified by the user to restrict the number of displayed fibers. These ROIs serve either as seed regions for the tracking ${ }^{13}$ or are used in the end to distinguish and display the relevant streamlines. ${ }^{14,15}$ Stopping criteria guarantee that tracking is aborted as soon as the streamline quits areas of anisotropic diffusion. Streamline propagation is performed by solving the streamline equation above. Discrete integration is thereby computed numerically using explicit or implicit integration schemes such as Euler or Runge-Kutta of different order.

The resulting streamlines provide a good impression of the spatial relation of fibers and anatomy and are therefore a valuable supplement for neurosurgical planning. However, even higher order integration does not prevent the streamlines from drifting away from the exact path due to numerical inaccuracies that accumulate during streamline propagation. Another limitation of fiber tracking is that crossing bundles in areas of planar anisotropy are not comprehensively represented. 


\section{DIRECTIONAL VOLUME GROWING}

To overcome the problems inherent in fiber tracking, a strategy for directional volume growing is presented which enables the extraction of separate tract systems. Directional volume growing is suited to get a more precise representation of the volume encompassed by white matter tracts and in addition allows to compare and estimate the quality of fiber tracking algorithms. Thereby, the entire volume potentially containing fibers is provided compared to fiber tracking which only shows a more restricted representation of the actual volume of interest. The algorithm for directional volume growing is compared to fiber tracking, both algorithms are described below.

Fiber Tracking The algorithm for streamline tracking works as follows: The user defines a number of thresholds such as minimum and maximum fiber length, a FA threshold for aborting streamline propagation and an angle threshold that prevents the streamline from sudden turns. Fiber tracking is started from ROIs defined by the user based on anatomical knowledge, provided that the respective FA value lies beyond the FA threshold. Streamline propagation is performed numerically by using a Runge-Kutta method of order four (RK4). If necessary, the local diffusion tensor is computed using trilinear interpolation for each tensor entry. The streamline propagation is aborted as soon as the local FA reaches a value below a predefined threshold or if the maximum fiber length is reached. In the case of multiple ROIs, only fibers crossing all ROIs are maintained.

Directional Volume Growing In general, volume growing algorithms start from a predefined seed region and spread out within the volume until some terminating criterion is reached. In addition, the presented directional volume growing takes into account the shape of the local tensor which controls the direction in which the process expands. The directional growing uses the same ROIs that were specified for fiber tracking. After starting from each voxel within the ROI the algorithm proceeds to neighboring voxels. In the same way as for fiber tracking, the growing process stops as soon as a FA value below a specified threshold is reached.

In the case of tensors corresponding to a cigar shaped ellipsoid (high value of linear diffusion $c_{l}$ ), directional volume growing expands the volume in the direction of the principal eigenvector. For this purpose the algorithm initially computes all vectors pointing from the center of the current voxel to the centers of the direct neighboring voxels. This set of vectors remains unchanged during the whole process. For each processed voxel the principal eigenvector is initially computed on the fly. A neighboring voxel is added to the grown volume if two threshold requirements are met: (1) The angle between the principal eigenvector and the vector pointing at the respective neighboring voxel must be below a specified threshold. (2) The FA value of the neighboring voxel must be above a predefined threshold. The angle $\alpha_{i}$ between the principal eigenvector $\vec{e}_{1}$ and the vector $\vec{x}_{i}$ pointing at neighboring voxel $i$ is computed according to

$$
\cos \alpha_{i}=\frac{\overrightarrow{e_{1}} * \overrightarrow{x_{i}}}{\left\|\overrightarrow{e_{1}}\right\| \cdot\left\|\overrightarrow{x_{i}}\right\|} .
$$

Figure 2 and the pseudocode give a schematical overview of the approach. For symmetry reasons, it is sufficient to check the angle for only 13 of the 26 neighboring voxels. If a neighboring voxel fulfills the angle threshold criterion, this neighboring voxel as well as its opposite voxel is appended to the volume.

In the planar case (high $c_{p}$ ) the algorithm tries to enlarge the grown volume in a plane according to the planar shape of the tensor. For this purpose the third eigenvector is used as normal vector $\vec{n}$ of the plane containing principal and second eigenvector since the eigenvectors of a symmetric tensor are orthogonal and form a Cartesian vector basis. To compute the angles between this plane and the vectors $\overrightarrow{x_{i}}$ pointing at the neighboring voxels, the angle between the normal $\vec{n}$ and $\overrightarrow{x_{i}}$ for each neighbor $i$ is computed. Note that 90 degrees have to be subtracted in order to obtain the angle between the plane and $\overrightarrow{x_{i}}$. In the same way as for linear diffusion, the associated neighboring voxel is added to the grown volume if this angle is below a specified threshold. 


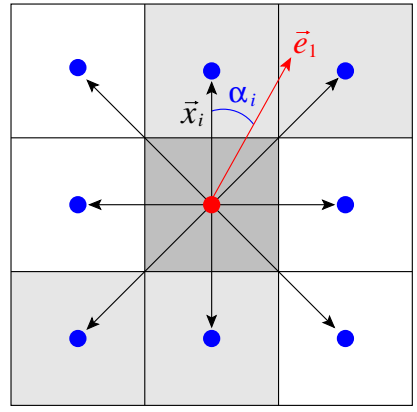

Figure 2. Schematical procedure for directional volume growing.

\author{
vol: Output voxels of directional volume growing \\ For all ROI voxels: \\ If ( FA( ROI voxel ) > FA_threshold ) \\ Add ROI voxel to vol ; \\ For (int $\mathrm{n}=0 ; \mathrm{n}<$ vol.dynamic_size; $\mathrm{n}++$ ): \\ If $\left(c_{l}>c_{p}, c_{s}\right)$ compute $\alpha_{i}$ using $\vec{e}_{1}, \overrightarrow{x_{i}}$; \\ If $\left(c_{p}>c_{l}, c_{s}\right)$ compute $\alpha_{i}$ using $\vec{n}, \overrightarrow{x_{i}}$; \\ If ( $\alpha_{i}<$ angle_threshold ) \\ If ( FA( neighbor_voxel_i ) > FA_threshold ) \\ If ( neighbor_voxel_i NOT IN vol) \\ Append neighbor_voxel_i to vol ;
}

\title{
5. RESULTS
}

DTI data was acquired and fiber tracking and directional volume growing were applied to 10 brain tumor cases. Based on anatomical knowledge, ROIs were manually defined which were used as starting regions for fiber tracking as well as for directional volume growing. The computation times for fiber tracking as well as directional volume growing are in the range of 40-60 seconds. All computations were performed on a standard PC (Intel Pentium 4, $2.4 \mathrm{GHz})$.

Figure 3 shows conventional volume growing (left) in comparison to iso-surface extraction based on FA values (right) for DTI data of a healthy volunteer. The fragmented nature of the iso-surface makes it difficult to clearly identify connectivity information which is important for surgery. In contrast, conventional volume growing produces a connected volume without any detached parts. The resulting visualization is further improved after iso-surface extraction based on the binary volume (middle).

Figure 4 shows two tumor patients. In both cases the pyramidal tract passes very close to the tumor. The streamline representation (left) already indicates a close spatial relation between tumor and fiber tract. Additionally, the directional volume growing (middle) clearly shows the volume occupied by white matter in the vicinity of the tumor. Therefore, there is a high possibility that the fiber tract is thicker than revealed by fiber tracking. A comparison of both representations (right) shows that the tracking algorithm computes streamlines which are completely encompassed by the suggested directional volume growing (in both cases the same FA threshold was applied). This observation indicates that there is no significant drift of the streamlines computed by the tracking algorithm. However, the volume derived by the presented growing procedure occupies more space than revealed by the streamlines. For tumor surgery this information is of high value since a more conservative estimate of the volume potentially containing fibers is provided.

\section{CONCLUSION}

Directional volume growing proved to be an adequate solution for estimating the entire volume occupied by white matter tracts within the human brain. In addition to conventional fiber tracking algorithms the presented strategy provides a more precise impression of the critical volume filled with white matter. Thereby, the possible location of fiber tracts is represented more comprehensively which is of high value for planning in neurosurgery.

\section{ACKNOWLEDGMENTS}

This work was supported by the Deutsche Forschungsgemeinschaft (German Research Foundation) in the context of SFB 603, Project C9 and the Graduate Research Center "3D Image Analysis and Synthesis". 


\section{REFERENCES}

1. E. Stejskal and J. Tanner, "Spin diffusion measurements: spin echoes in the presence of a time-dependent field gradient," J. Chem. Phys. 42, pp. 288-292, 1965.

2. G. Kindlmann, D. Weinstein, and D. Hart, "Strategies for direct volume rendering of diffusion tensor fields," IEEE Transactions on Visualization and Computer Graphics 6(2), pp. 124-138, 2000.

3. A. Aldroubi and P. Basser, "Reconstruction of vector and tensor fields from sampled discrete data," Contemp. Math. 247, pp. 1-15, 1999.

4. S. Pajevic, A. Aldroubi, and P. Basser, "A continuous tensor field approximation of discrete dt-mri data for extracting microstructural and architectural features of tissue," Journ. Magn. Reson. 54(1), pp. 85-100, 2002.

5. P. J. Basser and C. Pierpaoli, "Microstructural and physiological features of tissues elucidated by quantitative-diffusion-tensor mri," Journal of Magnetic Resonance, Series B 111(3), pp. 209-219, 1996.

6. D. L. Bihan, J.-F. Mangin, C. Poupon, C. A. Clark, S. Pappata, N. Molko, and H. Chabriat, "Diffusion tensor imaging: Concepts and applications," Journal of Magnetic Resonance Imaging 13, pp. 534-546, 2001.

7. C. Westin, S. Maier, H. Mamata, A. N. A, F. Jolesz, and R. Kikinis, "Processing and visualization for diffusion tensor MRI," Med Image Anal 6(2), pp. 93-108, 2002.

8. C. Pierpaoli, P. Jezzard, P. Basser, A. Barnett, and G. D. Chiro, "Diffusion tensor MR imaging of the human brain," Radiology 201(3), pp. 637-648, 1996.

9. F. H. Post, T. V. Walsum, F. J. Post, and D. Silver, "Iconic techniques for feature visualization," in Proc. IEEE Visualization, pp. 288-295, 1995.

10. F. Enders, S. Iserhardt-Bauer, P. Hastreiter, C. Nimsky, and T. Ertl, "Hardware-accelerated glyph based visualization of major white matter tracts for analysis of brain tumors," in Proc. SPIE Medical Imaging, 2005.

11. G. Kindlmann, "Superquadric tensor glyphs," in Proc. IEEE Visualization, 2004.

12. G. Kindlmann and D. Weinstein, "Hue-balls and lit-tensors for direct volume rendering of diffusion tensor fields," in Proc. IEEE Visualization, pp. 183-189, 1999.

13. L. Zhukov and A. Barr, "Oriented Tensor Reconstruction: Tracing Neural Pathways from Diffusion Tensor MRI," in Proc. IEEE Visualization, 2002.

14. P. Fillard, J. Gilmore, W. Lin, and G. Gerig, "Quantitative analysis of white matter fiber properties along geodesic paths," in Proc. MICCAI, 2003.

15. D. Akers, A. Sherbondy, R. Mackenzie, R. Dougherty, and B. Wandell, "Exploration of the brain's white matter pathways with dynamic queries," in Proc. IEEE Visualization, pp. 377-384, 2004. 

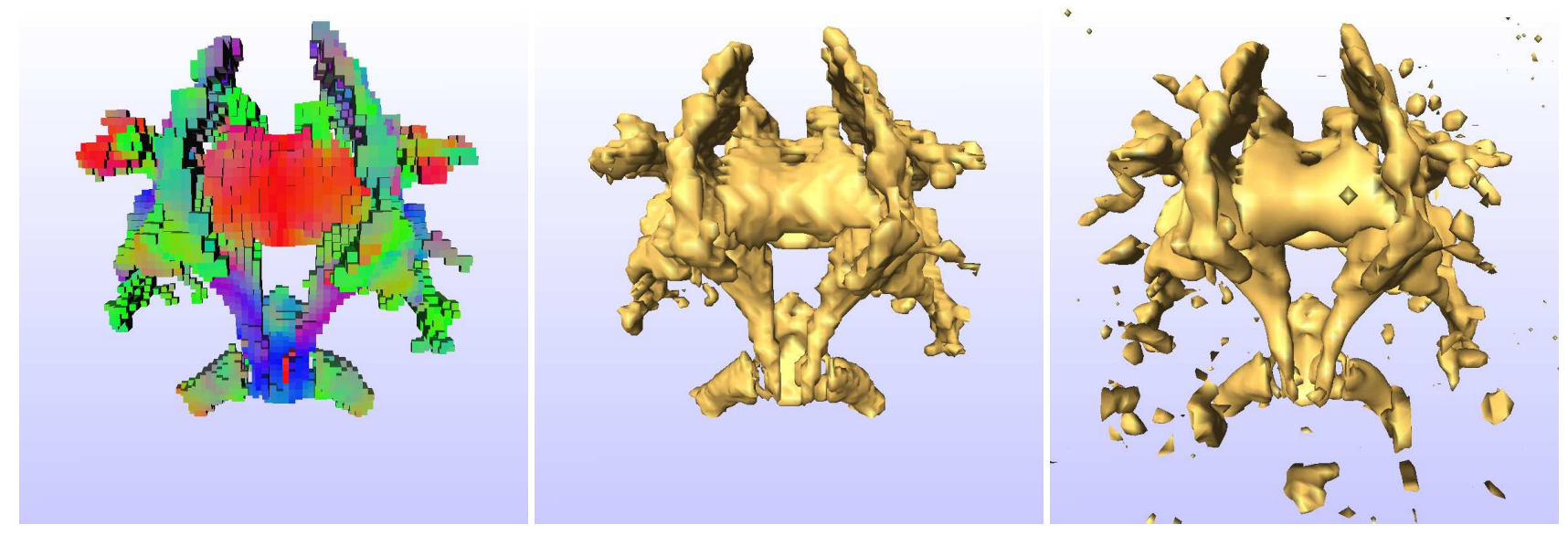

Figure 3. Conventional volume growing in healthy volunteer starting from a central seed region: Connected volume of high FA within the whole brain (left) and improved representation after iso-surface extracted of the binary volume (middle). Iso-surface extraction of the original FA dataset (right).
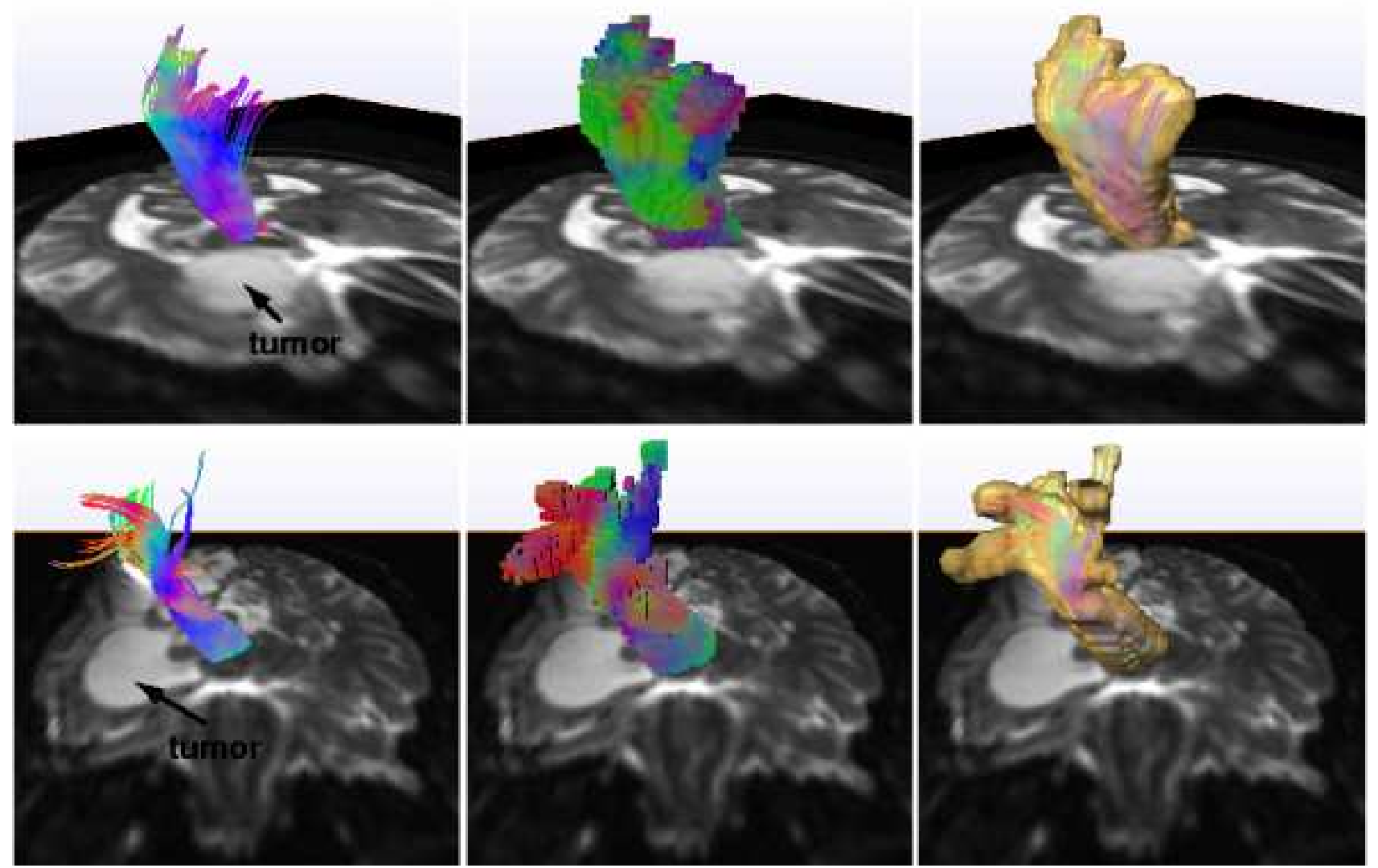

Figure 4. Visualization of pyramidal tract in two tumor patients (top/bottom) assigning RGB colors to the $\mathrm{x}, \mathrm{y}, \mathrm{z}-$ coordinates of the normalized principal eigenvector: Fiber tracking (left), directional volume growing showing binary volume (middle), overlay of fiber tracking and iso-surface derived from binary volume after directional volume growing (right). 